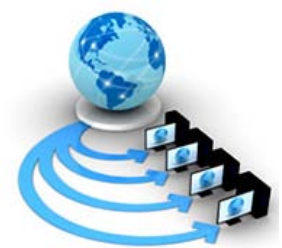

International Journal of Advanced Research in Computer Science

RESEARCH PAPER

Available Online at www.ijarcs.info

\title{
A NOBLE ENERGY SCAVENGING TECHNIQUE FOR WIRELESS BODY AREA NETWORK
}

\author{
Jobanpreet Kaur \\ Student at Department Of computer Science and \\ Engineering \\ Baba Banda Singh Bahadur Engineering College \\ Fatehgarh Sahib, Punjab,India
}

\author{
Er. Birinder Singh \\ Assistant Professor at Department of Computer Science and \\ Engineering \\ Baba Banda Singh Bahadur Engineering College \\ Fatehgarh Sahib, Punjab,India
}

\author{
Er. Jatinder Singh \\ Assistant Professor at Department of Computer Science and Engineering \\ Baba Banda Singh Bahadur Engineering College \\ Fatehgarh Sahib, Punjab,India
}

\begin{abstract}
Wireless Body Area Networks (WBAN) present a variety of Power Management challenges ranging from radio protocols, to node components. One of the most important constraints is the energy efficiency problem. This paper addresses the issue of power consumption in the field of Wireless Body Area Networks (WBAN). It presents a methodology used to perform real-time measurements of the energy consumption of a deployed WBAN. Body Area Networks (BANs) are widely used mainly for healthcare and fitness purposes. In both cases, the lifetime of sensor nodes included in the BAN is a key aspect that may affect the functionality of the whole system. Typical approaches to power management are based on a trade-off between the data rate and the monitoring time. The biggest challenge in use of WBAN is the battery power requirement.
\end{abstract}

Keywords: Wireless Body Area Networks (WBAN), Wireless Sensor Networks(WSN), Dynamic Power Management,Energy Efficiency,Energy Scavenging.

\section{INTRODUCTION}

A body area network (BAN), also referred to as a wireless body area network (WBAN) or a body sensor network (BSN), is a wireless network of wearable computing devices.A Wireless Body Area Network (WBAN) typically consists of a collection of low-power, miniaturised, invasive or noninvasive, lightweight devices with wireless communication capabilities that operate in the proximity of a human body. These devices can be placed in, on, or around the body, and are often wireless sensor nodes that can monitor the human body functions and characteristics from the surrounding environment [1]. The IEEE defines WBAN together with another network type near human body, the Wireless Personal Area Network (WPAN). The WBAN faces some technical problems that remain to be solved, one of which is saving energy in a device with limited battery life. This is a critical problem especially for an implanted device as it is impossible or very difficult to replace or recharge the battery. In order to have a long monitor time, it is mandatory to have long lived sensor nodes, and hence, power optimization at node level becomes equally important as well as power optimization at network level [2].

Energy-aware management is critical in order to increase the lifespan of sensor nodes [3]. A lot of research has been done to obtain a long-lived WBAN, where low power hardware is a fundamental requirement [4]. An overview of energy saving in wireless sensor networks has been established [5]. Much research has focused on reducing wireless communication power consumption and methods have been proposed [6][7].
Existing research suggests that shutting down processing units may optimize topology and communication range [8][9][10] . Network traffic and routing is exploited to decide when to turn the node into the sleeping state from an ON state and this has been shown to lower power consumption significantly [11]. However, topology control and energy aware routing protocols only reduce the transmission power of radio, and hence, are not suitable for low workload applications or the radio platforms with high idle power consumption. Sleep scheduling protocols can reduce the idle power consumption, however they are not effective with high network workloads or low radio idle power consumption. Although wireless communication is the major activity that consumes power for transmission, power consumption of microcontroller or processor units also have significant impact on energy budgets in WBAN nodes.

To achieve satisfactory network lifetime the problem of energy efficiency needs to be tackled on all levels of the entire network. Many researchers are devoted to reducing power consumption in various aspects of hardware design, data processing, network protocols and operating system [2]. Once the system has been designed, additional energy savings can be achieved by using dynamic power management (DPM), which shuts down the sensor node if no events occur [6]. The basic idea is to shut down devices when not needed and wake them up when necessary. This shutdown yields good savings. But while this power saving method seemingly provides significant energy gains, it is important to remember that sensor nodes communicate using short data packets. So we 
have to carefully implement Dynamic Power Management (DPM) to get the maximum life of sensor node.

\section{RELATED WORK}

Energy management can be addressed at several levels, from hardware to firmware, optimizing single components and subsystems, up to application of distributed power optimization strategies of systems such as wireless sensor networks. Presently, the trend in WBAN network is to improve the quality of service of the system which can be achieved by the efficient power consumption and battery management.The lifetime of a sensor network depends highly on the power consumption performed at each sensor node. A more efficient power management results in a longer network lifetime. Several methodologies have been proposed, accounts of the current energy conditions.

Recent research has enabled wireless sensor nodes to harvest energy from surrounding environments. An adaptive duty cycling algorithm that allows energy harvesting sensor nodes to autonomously adjust the duty cycle according to the energy availability in the environment is proposed in [12]. Prashanth [13] conducted studies about the steadiness measurements of the nodes powered by indoor solar system in the data queue. Due to the energy variation in the harvesting system, the throughput of the system also suffered degradation due to waiting time of data in queue. Some other facts are concluded such as: (i) the energy per byte is higher for low data rates as compared to higher data rates. (ii) the stability of the queue is maximum when data and energy follow an exponential distribution. The proposal of sleep-wake policies for energy harvesting sensor nodes results in the increased throughput and stabilization of queue [14].The data appearance rate, examination time and waiting time concludes the stability of the queue. Yang and Ulukus [15] designed a scheme to lessen the time interval to transmit the packets to the sink by adapting varying transmission rate according to the varying traffic load and available energy. Khairnar and Mehta [16] developed a transmission scheme to achieve a throughput close to the maximum achievable throughput for energy harvesting nodes. Depending upon the energy level and channel gain node adjusts its power by switching between only pre specified data rates. Murthy [17] proposed transmission schemes that transmit data by availing pre set modulation scheme for a specified data rate to achieve specific throughput and hence manage the data and power efficiently. Ozel et. al. in [18] developed adjusting transmission scheme in order to improve the throughput of the node. Energy is efficiently managed by varying the behavior of the transmitter according to the available energy. In above cited papers reveal the problems faced by energy harvesting system in WSN. As compared to the traditional WSN system, the quality of service is a more critical factor in WBAN system [19]. The work in [20] proposes an opportunistic classifier to optimize power consumption in a wearable movement monitoring system. In this case authors had the possibility to exploit features computed for the needs of the application. Energy is required to detect an event and transmit data, whereas both events require a specific amount of energy which is collected for some time. Hence the major challenge in energy harvesting $\mathrm{BN}$ is to keep both detector and transmitter in working state by providing the correct amount of energy at the correct time.

\section{SYSTEM MODEL}

\section{(a). WBAN Architecture}

The wireless body area network (WBAN) is very hot topic now days to monitor the health and movements of a patient using sensor nodes placed in or around the body. Sensors nodes are planted in the body tissue of the body are shown in Fig. 1. The main role of sensor nodes to send collected data to the main servers for evaluation of health of the patient and send collected data to the back end servers are that are connected with Wireless Local area networks (WLAN). Different types of signal are transmit from the nodes such as electrooculography (EOG), electromyography (EMG), electrocardiography (ECG), electroencephalography (EEG) also sense the temperature and conductance of the skin.

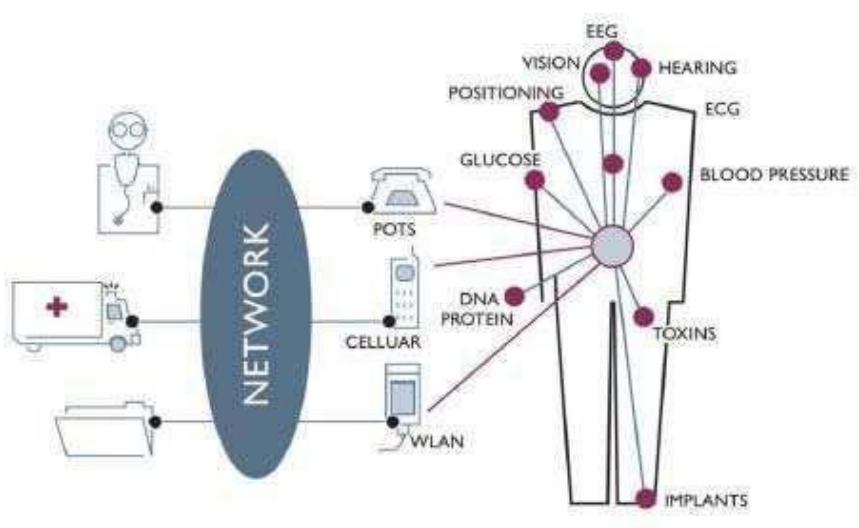

Figure 1: Wireless Body Sensor Network Model

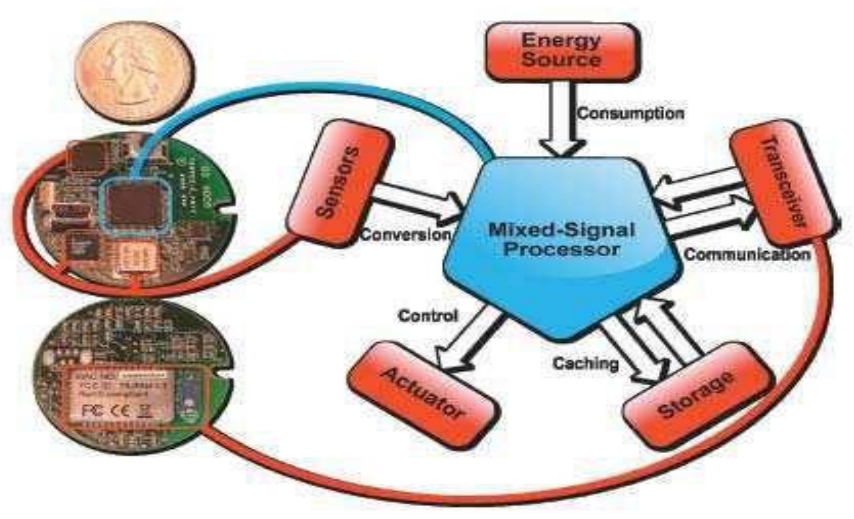

Figure. 2: WABNs node architecture

The main role of sensor nodes to send collected data to the main servers for evaluation of health of the patient and send collected data to the back end servers are that are connected with Wireless Local area net- works (WLAN).Different types of signal are transmit from the nodes such as electrooculography (EOG), electromyography Fig. 1.Wireless Body Sensor Network Model Fig. 2. WABNs node architecture(EMG),electrocardiography(ECG),electroencephal ography (EEG) also sense the temperature and conductance of the skin [21]. There are two types of devices that are used in WBAN one is sensors and other is actuators shows in Fig. 2. 
Function of sensors is to measure the different parameters of the body in different time places and send this data to actuators. These actuators send this data on the servers using WAN or different mediums [22].

\section{(b) Energy Efficiency in WBAN}

Energy-aware management is critical in order to increase the lifespan of sensor nodes. Energy consumption can be divided into three domains: sensing, (wireless) communication and data processing. WBAN we face different challenges such as weight of nodes, performance; most important is long battery life because nodes are powered with the battery. Also use different techniques to charges these batteries using different technologies [23]. Now a day's most of the research is to increase the battery timing and energy harvesting with low power consumption. A lot of research has been done to obtain a long-lived WBAN, where low power hardware is a fundamental requirement [24]. The wireless communication is likely to be the most power consuming. The power available in the nodes is often restricted. The size of the battery used to store the needed energy is in most cases the largest contributor to the sensor device in terms of both dimensions and weight. Batteries are, as a consequence, kept small and energy consumption of the devices needs to be reduced. In some applications, a WBAN's sensor/actuator node should operate while supporting a battery life time of months or even years without intervention. For example, a pacemaker or a glucose monitor would require a lifetime lasting more than 5 years. Especially for implanted devices, the lifetime is crucial. The need for replacement or recharging induces a cost and convenience penalty which is undesirable not only for implanted devices, but also for larger ones. The lifetime of a node for a given battery capacity can be enhanced by scavenging energy during the operation of the system. If the scavenged energy is larger than the average consumed energy, such systems could run eternally [25]. However, energy scavenging will only deliver small amounts of energy. A combination of lower energy consumption and energy scavenging is the optimal solution for achieving autonomous Wireless Body Area Networks. For a WBAN, energy scavenging from on-body sources such as body heat and body vibration seems very well suited.

The energy is a very crucial parameter in the WBAN systems. For instance, in invasive sensor nodes it is impossible to change the battery very frequently as it is very difficult to access the node. For non invasive nodes, if the system demands high battery consumption then it deters continuous monitoring and thus defeats the purpose. Again at transceiver part sending and routing contributes to maximum power consumption. If each node in the network transmit it the data directly to the sink node or base station, the energy consumed by the communication will be high. Thus Energy usage depends essentially on two things: Architecture of Sensor node and Network requirements. Architecture of the Sensor node is as depicted [26].Limited battery energy constraints can be overcome if the battery is recharged using energy scavenging and harvesting techniques. Much research has been proposed in order to develop an energy harvesting wireless sensor network, which seems to overcome the stringent power constraint problems. Recent research has enabled wireless sensor nodes to harvest energy from surrounding environments. An adaptive duty cycling algorithm that allows energy harvesting sensor nodes to autonomously adjust the duty cycle according to the energy availability in the environment is proposed in [24]. For a WBAN, energy scavenging from on-body sources such as body heat and body vibration seems very well suited.

\section{SIMULATION AND RESULTS}

In this paper, we have used the EXata simulation. It should be noted that the EXata simulation model has a lot of advantages compared to other simulation models such as NS2 and OPNET Modeler [27]. The virtual network models are used by EXata to simulate the behavior of the network under a wide variety of user-defined operating scenarios and application traffic patterns at real-time speeds and with real-world fidelity, accuracy and precision. Virtual network models created by network engineers with the EXata Developer platform can be leveraged by users of the Run-Time platform for ongoing analysis and experimentation.

\section{EXata Platform Architecture}

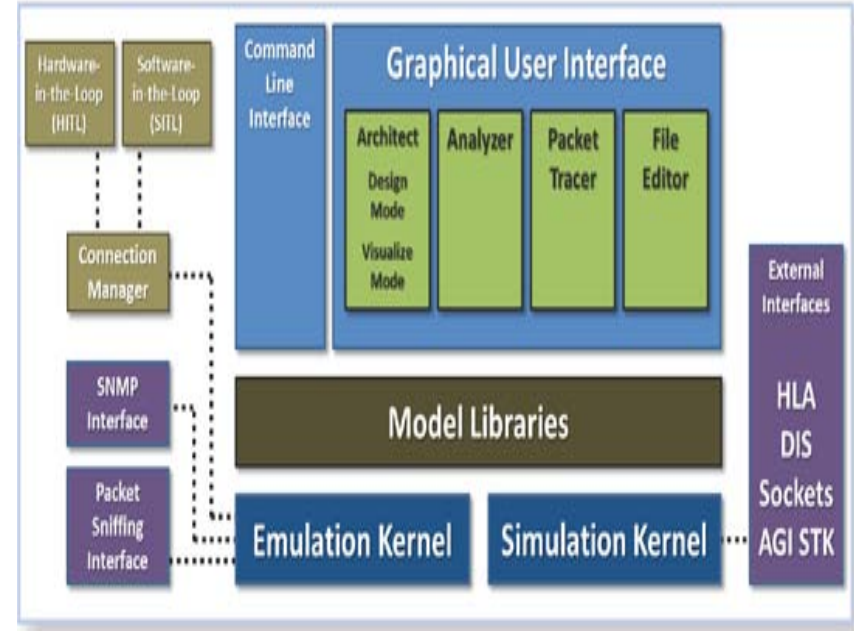

Figure 3: EXata Platform Architecture

\section{(a) Simulation}

\section{(i) Design Mode}

As proposed Exata based WBAN model in Figure 4, the architecture of a WBAN consists of a WBAN Coordinator and End Device sensor nodes. All of End Device nodes are directly cooperating with the WBAN Coordinator node. Nodes representing various types of network elements and endpoints are placed on a canvas. Each node is configured with wired or wireless network interfaces and communications characteristics. Nodes are then interconnected with the appropriate network links.The main objective of our work is to optimize energy consumption of the EXata based WBAN.Virtual network models can include subnets, mobility patterns of wireless users, other functional parameters of network nodes, and physical characteristics such as terrain and structures. A wide array of different application layer traffic and services that run on the network can be applied. 


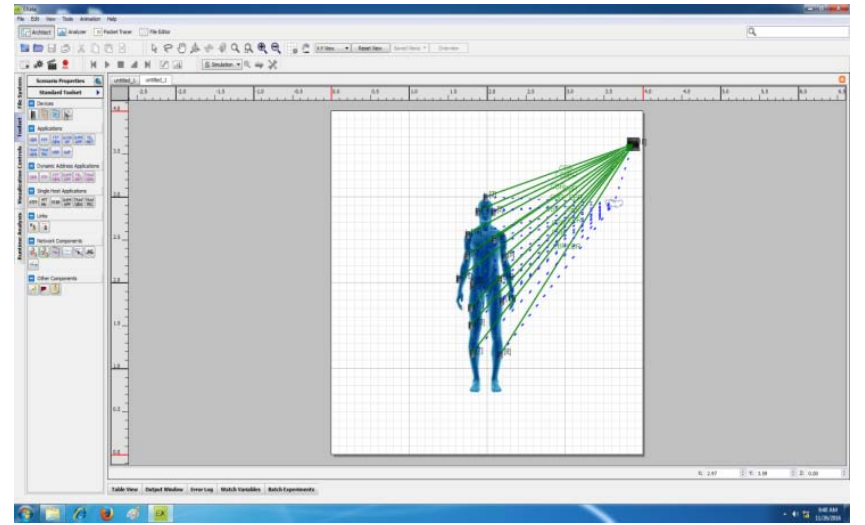

Figure. 4 : Exata Simulation for WBAN Model

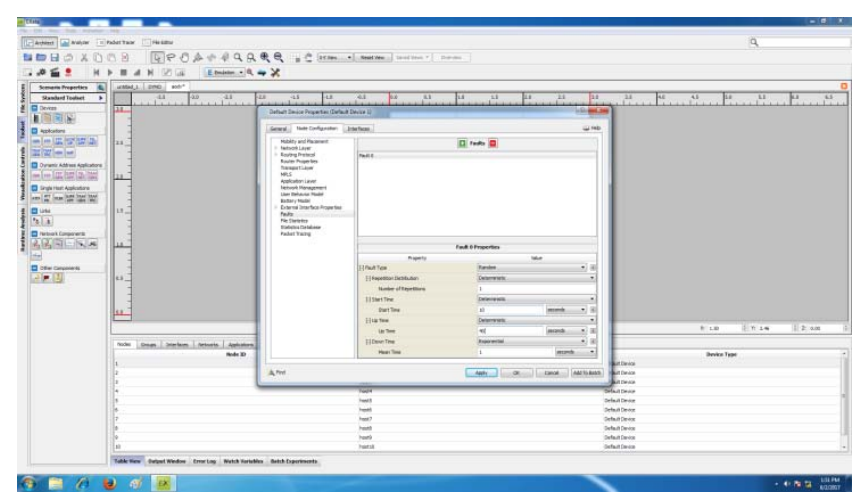

Figure 5: Configuration in EXata Platform

\section{(i) Visualize Mode}

Visualize Mode gives the user opportunities to perform indepth visualization and analysis of a network scenario created in Design Mode. As simulations are running, users can watch packets at various layers flow through the network and view dynamic graphs of critical performance metrics.

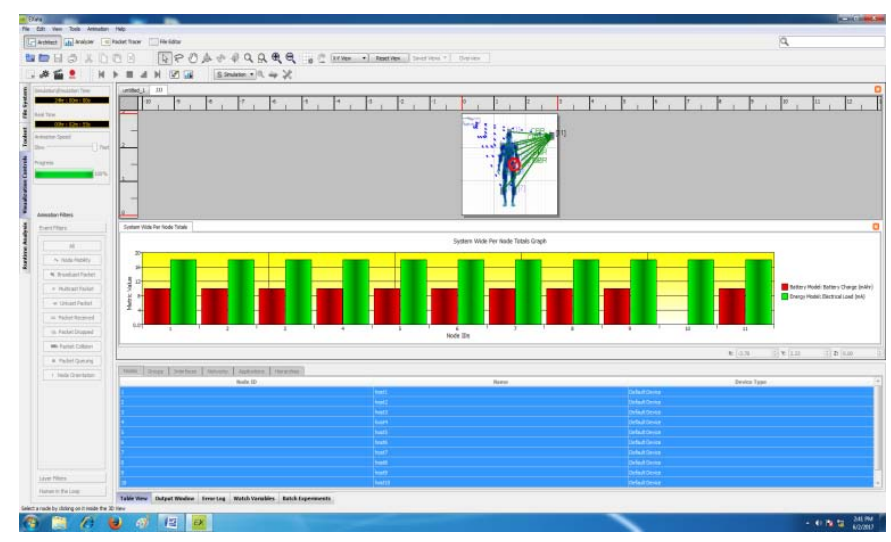

Figure 6: Visual Mode of EXata Scenario

\section{(i) Analyzer}

Analyzer is a statistical graphing tool that displays hundreds of metrics. You can customize the graph display. All statistics are exportable to spreadsheets in CSV format.

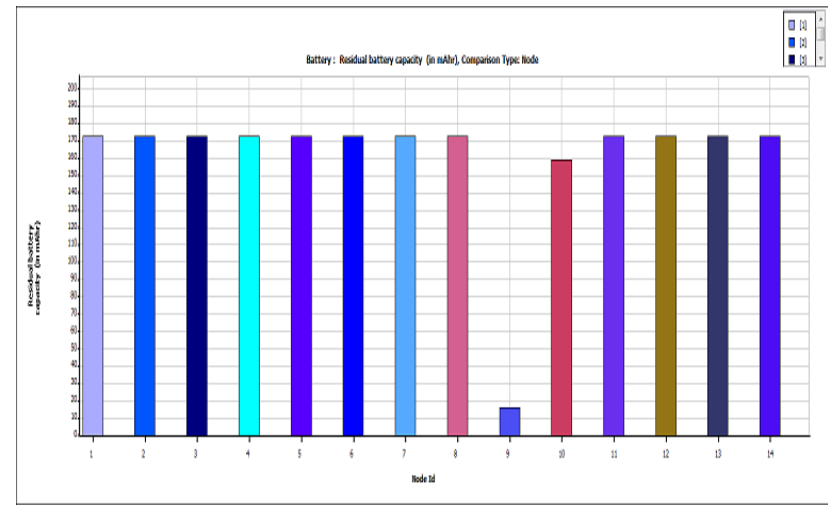

Figure 7. Residual battery capacity

(B) RESULT

Result show the comparison of energy consumed in two scenarios that was created in EXata. In Scenario 1 it shows the results where scenario is running for 24 hours. It takes more time and more energy consumption as compare to scenario 2.In scenario 2 we use a strategy of giving power supply when our system needs while in scenario 1 we give power supply all the time to perform its functionality. In scenario 2 we give power supply to system when it needs, For example in one hour a person walk for only 10 minute, in virtual world it needs power supply only for 10 minute for movement other 50 minute it does not need any power supply to movement, so when we give power supply for one hour it wasted, because our scenario needs power or energy inly for $10 \mathrm{~min}$ to movement.

\section{Energy Consumption}

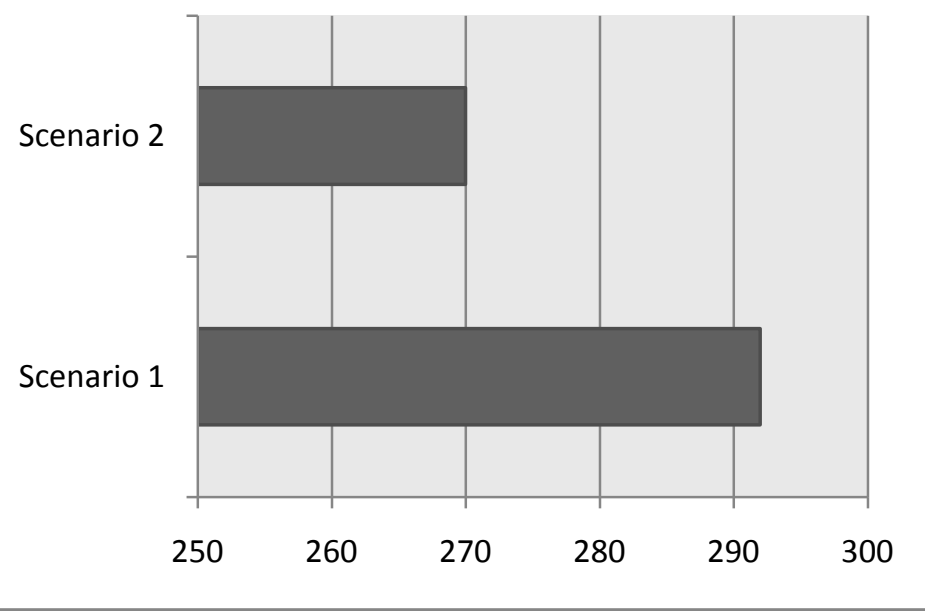

Figure 8: Comparison of scenarios based on energy consumption

So,We use a technique in scenario 2 in which we give power supply to scenario when it needs to perform its operation. In Scenario 2 we use the Dynamic Power Supply for perform its Functionality. Firstly System is in the sleep mode, if Some actions are perform by system it get entered into active mode and here we give it power supply when it is in active mode. So in scenario 2 energy consumption is less as compare to scenario 1. 


\section{CONCLUSIONS}

In this work we addressed the characterisation of the energy consumption in WBANs. Considering the resource limitation of WBAN's nodes and in order to further prolong the WBAN life, this method doesn't perform expensive processing computations and it efficiently manages the transmission power to further increase the communication reliability while avoiding spending unnecessary energy. Future work includes the integration of energy harvesters into our experimental setup and accurate battery level measurement techniques.

\section{REFERENCES}

[1] Cavallari.R, Martelli.F,Rosini.R,Buratti.C, Verdone.R, “A Survey on wireless Body Area Networks: Technologies and design challenges”, IEEE Communications surveys and tutorials,vol. 16, issue no. 3, pp. 1635-1657, 2014.

[2] B. H. Calhoun, D. C. Daly, N. Verma, D. Finchelstein, D. D. Wentzloff, A. Wang, S.-H. Cho, andA. P. Chandrakasan, "Design Considerations for Ultra-low Energy Wireless Microsensor Nodes," IEEE Transactions on Computers, June, 2005.

[3] I. F. Akyildiz, W. Su, Y. Sankarasubramaniam, and E. Cayirci, "A survey on sensor networks," Communications Magazine, IEEE, vol. 40,no. 8, pp. 102-114, August, 2002

[4] J. Hill, M. Horton, R. Kling, and L. Krishnamurthy, "The platforms enabling wireless sensor networks," Communications of the ACM, vol. 47, no. 6, pp. 41-46, June, 2004.

[5] X. Cui, X. Zhang, and Y. Shang, "Energy-saving strategies of wireless

sensor networks," in Proc. of Int. Symposium on Microwave, Antenna,

Propagation and EMC Technologies for Wireless Communications, pp.178-181, August 16-17, 2007.

[6] I. Slama, B. Jouaber, and D. Zeghlache, "Optimal power management scheme for heterogeneous wireless sensor networks: Lifetime maximization under qos and energy constraints," in Proc. of 27th Int.

Conf. on Distributed Computing Systems Workshops, pp. 6974, June 19-25, 2007

[7] A. S. Zahmati, N. M. Moghadam, and B. Abolhassani, "Epmplcs: An efficient power management protocol with limited cluster size for wireless sensor networks," in Proc. of 27th Int. Conf. on Distributed Computing Systems Workshops, pp. 69-72, June 22-29, 2007.

[8] H.-C. Jang and H.-C. Lee, "Efficient energy management to prolong wireless sensor network lifetime," in Proc. of 3rd IEEE/IFIP Int. Conf. in Central Asia on Internet, pp. 1-4, September, 2007.

[9] C. Lin, Y.-X. He, and N. Xiong, "An energy-efficient dynamic power management in wireless sensor networks," in Proc. of 5th Int. Symposium on Parallel and Distributed Computing, pp. 148-154, 2006.

[10] X. Wang, J. Ma, and S. Wang, "Collaborative deployment optimization and dynamic power management in wireless sensor networks," in Proc.

of 5th Int. Conf. on Grid and Cooperative Computing, pp. 121128, 2006.

[11] H. Wang, W. Wang, D. Peng, and H. Sharif, "A route-oriented sleep approach in wireless sensor network," in Proc. of the Int. Conf. on

Communication Systems, pp. 1-5, 2006.

[12] N. Dang, E. Bozorgzadeh, and N. Venkatasubramanian, "Quares: Quality-aware data collection in energy harvesting sensor networks," in
Proc. of 2011 Int. Green Computing Conf. and Workshops, pp. 1-9, July25-28, 2011.

[13] V.Prashanth,T.V.Prabakar,K.Prakaruthi,H.S.Jamadgani," Queue Stability Measurements for Energy Harvesting Sesnsor Nodes', in Proceedings of National Conference on Communications'”(NCC),pp.1-5,Feburary 2012.

[14] V, Joseph,V Sharma and U Mukherji,'’Optimal Sleep Wakeup Policies for Energy Harvesting Communication System"'pp.1-6,14-18 June 2009.

[15] J.Yang,S.Ulukus,' Optimal Packet Scheduling in an Energy Harvesting Communication System',Journal IEEE Transactions on Communications,(ICC), pp. 220230,Janyary 2012.

[16] P.S.Khairnar and N.B.Mehta,"Power and Discrete Rate Adaption for Energy Harvesting Wireless Sensor Networks", in the Proceedings of IEEE International Conference on Communication,pp. 1-5, June 2011.

[17] C.R.Murthy,'”Power Management and Data Rate Maximization in Wireless Energy Harvesting Sensors", in the Proceedings of 19th International Symposium on Personal Indoor and Mobile Radio Communication,pp. 15,Sep 2008.

[18] O.Ozel,K.Tutuncuglo,J.Yang ,S.Uulukus and A.Yener,'Adaptive Transmission Policies for Energy Harvesting Wireless Nodes in the Fading Channels', in the Proceedings of 45th Annual Conferenece on Information Sciences and Systems,pp.1-6,March 2011.

[19] M.A.Ameen,A.Neesa,KS.Kwak,”QOS Issues with Focus on Wireless Body Area Networks" in Proceedings of 3rd International Conference on Convergence and Hybrid Information Technology,pp. 801-807,November 2008.

[20] V. Raghunathan, S. Ganeriwal, and M. Srivastava, "Emerging techniques for long lived wireless sensor networks," Communications Magazine,IEEE, vol. 44, no. 4, pp. 108114, 2006.

[21] . L. Huang, M. Ashouei, R. F. Yazicioglu, J. Penders, R. J. Vullers, G. Dolmans, P. Merken, J. Huisken, H. de Groot, C. Van Hoof, et al., "Ultra-low power sensor design for wireless body area networks- challenges, potential solutions, and applications.” JDCTA, Vol. 3, No. 3, pp. 136-148, 2009.

[22]. B. Latre', B. Braem, I. Moerman, C. Blondia, and P. Demeester, "A survey on wireless body area networks," Wireless Networks, Vol. 17, No. 1, pp. 1-18, 2011.

[23]. D. T. H. Lai, M. Palaniswami, and R. Begg, Healthcare Sensor Net- works: Challenges toward Practical Implementation. CRC Press, 2011.

[24] G. Anastasi, M. Conti, M. Di Francesco, and A. Passarella, "Energy conservation in wireless sensor networks: A survey,” IEEE,vol. 7, pp. 537-568.

[25] Elias and A. Mehaoua, "Energy-aware topology design for wireless body area networks,” in IEEE(2012), pp. 34093410.

[26] K. Pervez, Md. Asdaque Hussain, K.S. Kyung, "Medical Applications of Wireless Body Area Networks," in international Journal of Digital Content Technology and its Applications, vol. 3,issue no. 3, pp. 185-193, 2009.

[27 ] M. Chen, "Opnet network simulation," Press of TsinghuUniversity vol. 1, 2004.

[28] Divjot Kaur, R S Uppal , Jatinder Singh saini, Energy Efficient Data Collection in WSN with Multi-Hop Routing, International Journal of Computer science Enginnering and Technology, vol: 6, no: 12, pp: 149-152, 2016.

[29] Kaur. J, Singh. Er, Birinder Singh,“A Survey on Wireless Body Area Network Challenges and Energy Management Issues”, IJESC(2017), Vol. 7, No.5 , pp. 11581- 11585. 